\title{
ON THE NONSIMPLICITY OF SOME CONVERGENCE CATEGORIES
}

\author{
E. LOWEN AND R. LOWEN
}

(Communicated by Dennis Burke)

\begin{abstract}
The category TOP of topological spaces is known to be Convsimple in the sense that there exists a single object $E$ in Top such that Top is the epireflective hull of $\{E\}$ in the category Conv of convergence spaces. Prtop, the category of pretopological spaces is also Conv-simple. We show that on the contrary the category Pstop of pseudo topological spaces and Conv itself are not Conv-simple. More specifically every epireflective subcategory of Conv which contains all Hausdorff $c$-embedded locally compact spaces is not Conv-simple.
\end{abstract}

For references on topological categories, reflective subcategories and reflective hulls we refer to $[6,8,9,10]$.

Let $\mathscr{A}$ be a topological category.

All subcategories $\mathscr{B}$ are assumed to be full and isomorphim-closed.

A subcategory $\mathscr{B}$ of $\mathscr{A}$ is epireflective in $\mathscr{A}$ if it is closed with respect to the formation of products and subobjects in $\mathscr{A}$. In this context " $Y$ is a subobject of $X$ " means that there exists an embedding from $X$ to $Y$. This notion coincides with the categorical notion of extremal subobject.

Every subcategory $\mathscr{E}$ of $\mathscr{A}$ is contained in a smallest epireflective subcategory, its epireflective hull, which is denoted by $R \mathscr{E}$. An object $A$ of $\mathscr{A}$ belongs to $R \mathscr{E}$ if and only if $A$ is a subobject of a product of objects of $\mathscr{E}$. A subcategory $\mathscr{B}$ of $\mathscr{A}$ is called $\mathscr{A}$-simple if there exists a single object $E$ of $\mathscr{B}$ such that $\mathscr{B}$ is the epireflective hull in $\mathscr{A}$ of the class $\{E\}$, i.e., $\mathscr{B}=R\{E\}$.

Several examples of this situation are well known. If we take $\mathscr{A}=$ Conv then its bireflective subcategory Top is Conv-simple. Several subcategories of TOP are Conv-simple too, see for instance $[4,5,6,7,9,14]$. Simplicity remains true if TOP is enlarged to the bireflective subcategory Prtop. This can be derived from results in [1].

In this paper we show that simplicity however does not extend to the larger bireflective subcategories Pstop or to Conv itself.

Received by the editors December 4, 1987 and, in revised form, April 11, 1988.

1980 Mathematics Subject Classification (1985 Revision). Primary 18B99, 54B30, 54A25.

This paper was presented at the conference "Convergence structures in topology and analysis" at Mathematiches Forschungs Institute Oberwolfoch, December 1987. 
For an arbitrary convergence space $Y$ we construct a Hausdorff, locally compact, $c$-embedded convergence space $X$ such that for any source $\left(f_{i}: X \rightarrow Y\right)_{i \in I}$ the space $X$ is not initial in Conv. It follows that every epireflective subcategory of Conv containing all Hausdorff, locally compact, $c$-embedded spaces, is not Conv-simple.

For notions about Conv we refer to the basic literature $[2,3,11,12,13]$.

The following set theoretical property is fundamental for our results. We use the following notations. If $X$ is a set and $\mathscr{U}$ is an ultrafilter on $X$ then

$$
\|\mathscr{U}\|=\min \{\operatorname{card} U \mid U \in \mathscr{U}\} .
$$

An ultrafilter $\mathscr{U}$ on $X$ is uniform if $\|\mathscr{U}\|=\operatorname{card} X$. If $\mathscr{B}$ is a filterbase on $X$ then the filter generated on $X$ is denoted by stack $\mathscr{B}$. If $B$ is a subset of $X$ then the filter generated by $\{B\}$ is denoted by $\operatorname{stack}_{X} B$. Such filters are called principal filters.

Proposition. If $X$ and $Y$ are sets, $X$ is infinite and $\operatorname{card} X>\operatorname{card} Y$, if $\mathscr{U}$ is a uniform ultrafilter on $X$ and $f$ is a function from $X$ to $Y$ then there exists a uniform ultrafilter $\mathscr{W}$ on $X$ different from $\mathscr{U}$ such that

$$
\operatorname{stack}_{Y} f(\mathscr{W})=\operatorname{stack}_{Y} f(\mathscr{U}) .
$$

Proof. Let $X, Y, \mathscr{U}$ and $f$ be as in the assertion above. We can choose a set $U \in \mathscr{U}$ such that $\operatorname{card} U=\|\mathscr{U}\|$ and $\operatorname{card} f(U)=\left\|\operatorname{stack}_{Y} f(\mathscr{U})\right\|$. Let

$$
\mathscr{P}=\left\{f^{-1}(y) \mid y \in f(U)\right\}
$$

and consider the following subcollections

$$
\begin{aligned}
& \mathscr{P}_{1}=\{P \in \mathscr{P} \mid \operatorname{card}(P \cap U)<\omega\} \\
& \mathscr{P}_{2}=\{P \in \mathscr{P} \mid \operatorname{card}(P \cap U) \geq \omega\} .
\end{aligned}
$$

Notice that $\mathscr{P}_{2}$ is not empty. Indeed suppose that $\mathscr{P}=\mathscr{P}_{1}$. If card $\mathscr{P}_{1}<\omega$ it follows that card $U$ is finite. If card $\mathscr{P}_{1} \geq \omega$ then

$$
\begin{aligned}
\operatorname{card} U & =\max \left\{\operatorname{card} \mathscr{P}_{1}, \sup _{P \in \mathscr{P}_{1}} \operatorname{card}(P \cap U)\right\} \\
& =\operatorname{card} \mathscr{P}_{1}=\operatorname{card} f(U) .
\end{aligned}
$$

Thus in both cases we have a contradiction. Now for every $P \in \mathscr{P}_{2}$ choose disjoint sets $A_{P}$ and $B_{P}$ such that $P \cap U=A_{P} \cup B_{P}$, and $\operatorname{card} A_{P}=\operatorname{card} B_{P}=$ $\operatorname{card}(P \cap U)$.

Further, let

and

$$
A:=\left(\bigcup_{P \in \mathscr{P}_{1}} P \cap U\right) \cup\left(\bigcup_{P \in \mathscr{P}_{2}} A_{P}\right)
$$

$$
B:=\left(\bigcup_{P \in \mathscr{P}_{1}} P \cap U\right) \cup\left(\bigcup_{P \in \mathscr{P}_{2}} B_{P}\right) \text {. }
$$


Since $A \cup B=U$ one of the sets $A$ or $B$ belongs to $\mathscr{U}$. Suppose $B \in \mathscr{U}$. Let $g: B \rightarrow A$ be any function such that $g(x)=x$ if $x \in \bigcup_{P \in \mathscr{P}_{1}} P \cap U$ and such that $g$ maps $B_{P}$ bijectively onto $A_{P}$ if $P \in \mathscr{P}_{2}$. Then clearly

$$
g(B) \cap B=\bigcup_{P \in \mathscr{P}_{1}} P \cap U
$$

and thus considering as before cases as to whether card $\mathscr{P}_{1}<\omega$ or card $\mathscr{P}_{1} \geq \omega$ one finds

$$
\operatorname{card}(g(B) \cap B)<\operatorname{card} U
$$

from which it follows that $g(B) \notin \mathscr{U}$. Put

$$
\mathscr{W}=\operatorname{stack}_{X}\{g(V \cap B) \mid V \in \mathscr{U}\} .
$$

Then $\mathscr{W}$ is a uniform ultrafilter on $X$, different from $\mathscr{U}$. On the other hand, by construction we have

$$
\operatorname{stack}_{Y} f(\mathscr{W})=\operatorname{stack}_{Y} f(\mathscr{U}) .
$$

Theorem. For every convergence space $Y$ there exists a Hausdorff locally compact c-embedded convergence space $X$ such that for any source $\left(f_{i}: X \rightarrow Y\right)_{i \in I}$ in Conv the space $X$ is not initial.

Proof. Let $Y$ be an arbitrary convergence space. Take an infinite set $X$ with cardinality strictly larger than the cardinality of the underlying set of $Y$. Further we fix a point $a \in X$ and a uniform ultrafilter $\mathscr{U}$ on $X$. We make $X$ a pseudotopological space by defining the following convergent ultrafilters:

(1) if $x \neq a$ then an ultrafilter $\mathscr{W}$ converges to $x$ if and only if $\mathscr{W}=\operatorname{stack}_{x}\{x\}$

(2) an ultrafilter $\mathscr{W}$ converges to $a$ if and only if $\mathscr{W}=\operatorname{stack}_{x}\{a\}$ or $\mathscr{W}$ is nonprincipal and $\mathscr{W} \neq \mathscr{U}$.

$X$ clearly is Hausdorff and locally compact and since every convergent nonprincipal ultrafilter satisfies $\overline{\mathscr{W}}=\mathscr{W} \cap \operatorname{stack}_{x}\{a\}$ it follows that $X$ is regular. The pretopological reflection of $X$ is a compact Hausdorff topological space. It follows that the pretopological reflection of $X$ coincides with the completely regular reflection. Hence $X$ is also $\omega$-regular, and thus $c$-embedded. Now let $\left(f_{i}: X \rightarrow Y\right)_{i \in Y}$ be any source in Conv. From the proposition it follows that $\mathscr{U}$ converges to $a$ in the initial structure of the source. Hence $X$ is not initial.

Corollary. Every epireflective subcategory of Conv containing all Hausdorff locally compact c-embedded spaces is not Conv-simple.

In particular the previous result can be applied to conclude that the following epireflective subcategories of Conv are not Conv-simple: Conv itself, Pstop, the categories $T_{1}$ Conv, $T_{1}$ Pstop, HConv, HPstop, the categories of all $c$ embedded spaces, of all $\omega$-regular spaces and the category of all regular spaces. 


\section{REFERENCES}

1. G. Bourdaud, Espaces d'Antoine et Semi Espaces D'Antoine, Cahiers Topologie Géom. Différentielle XVI 2 (1975).

2. G. Choquet, Convergences, Ann. Univ. Grenoble, Sect. Sci. Math. Phys. 23 (1948), 57-112.

3. H. Fisher, Limesraüme, Math. Ann. 137 (1959), 260-303.

4. D. Hajek and A. Mysior, On non-simplicity of topological categories, Lecture Notes in Math, vol. 719, Springer-Verlag, Berlin and New York, 1979, pp. 84-93.

5. D. Hajek and R. Wilson, The non-simplicity of certain categories of topological spaces, Math. Z. 131 (1973), 357-359.

6. H. Herrlich, Topologische Reflexionen und Coreflexionen, Lecture Notes in Math., no. 78, Springer-Verlag, Berlin and New York, 1968.

7. __ Categorical topology, Gen. Top. Appl. 1 (1971), 1-15.

8. __ Topological structures, Math. Centre Tracts 52, Amsterdam (1974), 59-122.

9. __ Categorical Topology 1971-1981, Proc. of the Fifth Prague Symposium 1981, Heldermann Verlag, Berlin, 1983, pp. 279-383.

10. H. Herrlich and G. Strecker, Category theory: an introduction, 2nd ed. Sigma Series Pure Math., Vol. 1, Heldermann Verlag, Berlin, 1979.

11. D. Kent and G. Richardson, Locally compact convergence spaces, Michigan Math. J. 22 (1975), 353-360.

12. D. Kent, K. McKennon, G. Richardson, and M. Schroder, Continuous convergence in $C(X)$, Pacific J. Math. 52 (1974), 271-279.

13. H. Kowalsky, Limesraüme und Komplettierung, Math. Nachr. 12 (1954), 301-340.

14. T. Marny, Rechts-Bikategoriestrukturen in topologischen Kategorien, Thesis, F. U. Berlin.

Departement Wiskunde, Vrije Universiteit Brussel, Pleinlaan 2, B-1050 Brussel

Wiskundige ANalyse, Universiteit ANTwerpen, R.U.C.A., GroenenborgerlaAn 171, B-2020 ANTWERPEN 